EPJ Web of Conferences 64, 04001 (2014)

DOI: $10.1051 /$ epjconf/ 20146404001

(C) Owned by the authors, published by EDP Sciences, 2014

\title{
The Early History of Stellar Spin: the Theory of Accretion onto Young Stellar Objects
}

\author{
Ralph E. Pudritz ${ }^{1,2, a}$ and Sean Matt ${ }^{3, b}$ \\ ${ }^{1}$ Dept. of Physics and Astronomy, McMaster University, Hamilton, ON L8S 4M1, Canada \\ ${ }^{2}$ Origins Institute, McMaster University, ABB 241, Hamilton, ON L8S 4M1, Canada \\ ${ }^{3}$ Physics and Astronomy, University of Exeter, Physics Building, Stocker Road, Exeter, U.K. EX4 4QL
}

\begin{abstract}
The interaction of the magnetospheres of forming stars with their surrounding protostellar disks results in magnetospheric accretion flow onto the star. How is the associated angular momentum of accreting material channelled? The resolution of this issue is crucial for understanding the origin of the spins of pre main sequence stars. A significant fraction of these rotate very slowly, which indicates that an efficient angular momentum transport mechanism is at work to counteract the strong accretion spin up torques. We review the observational, theoretical, and computational advances in the field and argue that an accretion powered stellar winds together with highly time variable mass ejections from the disk/magnetosphere interface is a likely solution.
\end{abstract}

\section{Introduction}

Stars formation involves the accretion of gas through protostellar disks. Disks themselves arise as a consequence of the gravitational collapse of dense, rotating magnetized "cores" in molecular clouds. The cores out of which disks form contain $10^{4}$ orders of magnitude more specific angular momentum than will the resulting pre main sequence (PMS) star. While most of this is shed by spiral waves, turbulent disk viscosity and disk winds in and from the disk, the fate of specific angular momentum as gas accretes onto the star is crucial for predicting the early spin history of PMS.

For low mass stars at least, there is abundant evidence that the disk is truncated by a protostellar magnetosphere and that accretion flow occurs along magnetic field lines that connect it with the surface of the star. For observed accretion rates onto the star of $10^{-7} M_{\odot} y r^{-1}$, and knowing the specific angular momentum of material at the inner edge of the Keplerian disk, it is easy to compute the rate at which such a flow would deliver angular momentum to the young star. The answer is surprising stars without any other counteracting torque would spin up to break up speed within $10^{5}$ years, much shorter than the lifetimes of accretion disks (typically $3-5 \times 10^{6} \mathrm{yrs}$ ). Stellar spins have a wide scatter in spin period, but a large fraction of PMS rotate very slowly [2]. Why does this occur? The solution of this problem has occupied astrophysicists for decades. The revolution in our ability to measure stellar spins and magnetic fields, and the advent of new theoretical models and simulations have turned this into a forefront problem in star formation studies. This review focuses on the question of how does disk/magnetosphere interaction resolves the stellar angular momentum problem.

\footnotetext{
a e-mail: pudritz@physics.mcmaster.ca

be-mail: S.Matt@exeter.ac.uk
} 


\section{Magnetospheric Accretion}

Observational studies of the spins of stars now cover a broad range of stellar evolution from 1 Myr to 1 Gyr. Over the last seven years, more than 5000 new measurements of stellar rotations have been made [3]. A key finding that supports earlier work is that young, low mass $\left(0.1-1 M_{\odot}\right)$ pre main sequence (PMS) stars that are a few Myr old span a broad range of stellar spins with most ranging from periods of 1 - 10 days. In figure 1, the older data already showed another important trend; namely that more than half of the T Tauri stars are slow rotators. For typical TTS stars with masses $M_{*}=0.5 M_{\odot}$, radii $R_{*}=2 R_{\odot}$, and a period of 8 days, the ratio $\mathrm{f}$ of the observed spin period to the break up spin rate is $f<0.1$. This value is maintained despite high accretion rates onto these stars.

Early observations of $\mathrm{H} \alpha$ and other lines emitted from accreting T Tauri stars (TTS) by were interpreted as indicative of massive stellar winds [5]. Subsequent work however showed that these lines were better understood as arising from magnetospheric accretion $[4,6]$. The argument for accretion was that the UV excess of TTS can be understood as emission from accretion shocks. Red shifted absorption also had a natural explanation in this picture as does variability, which could arise in part due to a non-axisymmetric magnetic field and stellar rotation.

The basic elements of a highly idealized picture are sketched in figure 2. A dipolar magnetic field that is aligned with the stellar spin axis is sufficiently strong to truncate the disk out to a number of stellar radii. This truncation radius $R_{t}$ is where the stellar magnetospheric stress on the disk exceeds its own internal turbulent Reynold's stress (this is often described as the point where the magnetic pressure of the magnetosphere balances the gas pressure of the surrounding gas). At this radius, the gas is slowed down from its Keplerian value to the stellar angular velocity $\Omega_{*}$ and therefore goes into free-fall to the star along the field lines [7-9]. This truncation radius depends upon the accretion rate $\left(\dot{M}_{a}\right)$ and the strength of the stellar dipole field through the magnetic dipole moment $\mu$; and is expressed as $R_{t}=\left(2 \gamma_{c}\right)^{2 / 7}\left(G M_{*}\right)^{-1 / 7}\left(\dot{M}_{a}\right)^{-2 / 7} \mu^{4 / 7}$ where $\gamma_{c} \simeq 1$. The median value of the disk accretion rate in CTTS is $\sim 10^{-8} M_{\odot} y r^{-1}$ but with a broad dispersion of an order of magnitude [10].

The link between the theoretical picture of accretion flow onto the star and the structure of the different observed emission lines that are the diagnostics are illustrated in figure 3 . The peak of the line is from matter leaving the disk at $v \simeq 0$. This emission comes from a large emitting volume. The wings arise from the high speed gas emitted by gas that reaches the photosphere at a free fall speed of

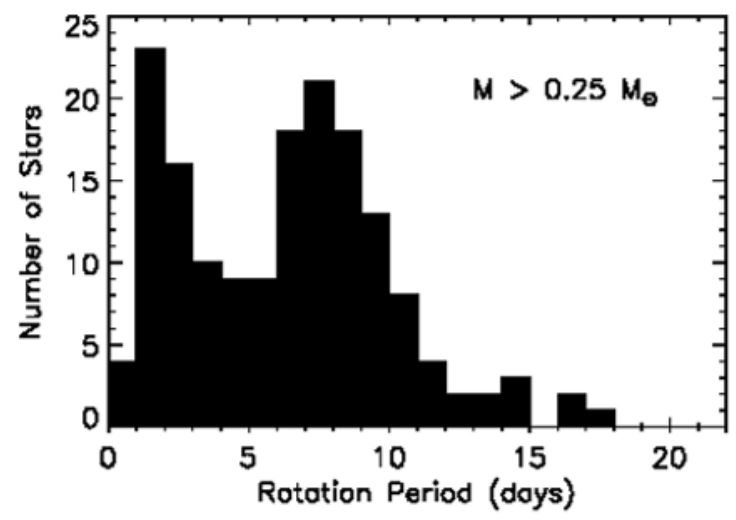

Figure 1. The distribution of stellar rotation periods of PMS. Adapted from [1]. 


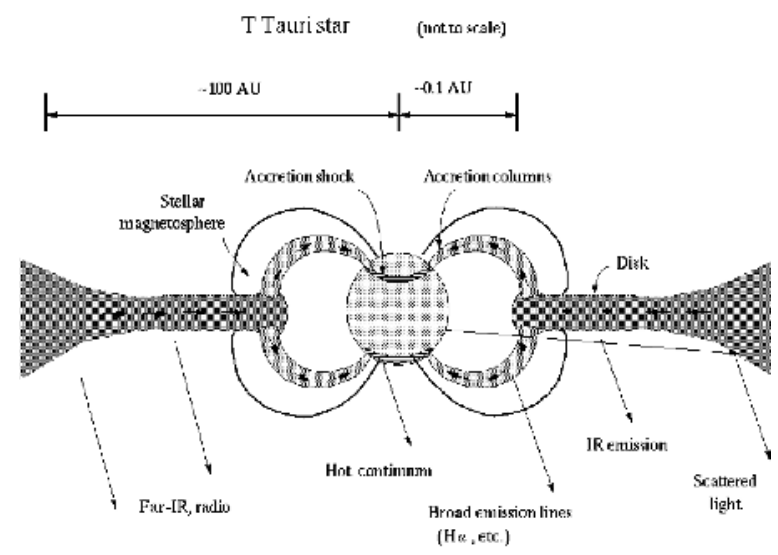

Figure 2. Magnetospheric accretion onto young stellar objects with a dipole magnetic field. The star's magnetic field is strong enough to disrupt the disk, at which point it undergoes accretion onto the central star. After L. Hartmann.

hundreds of $\mathrm{km} / \mathrm{s}$. The blue shifted material in the line wings is from material that is moving towards the star and the observer. If the observer's line of sigh crosses material in front of the shock, then a redshifted component is seen at $v \simeq v_{f f}$. The fit of this model to the observations is very good [4]. The gas slows down to merge with the photosphere through the accretion shock. The shock releases $\mathrm{X}$-rays which heat the pre shock material above it, as well as the photosphere below. The heated material then cools by radiating energy at short (UV) wavelengths as it radiates a substantial fraction
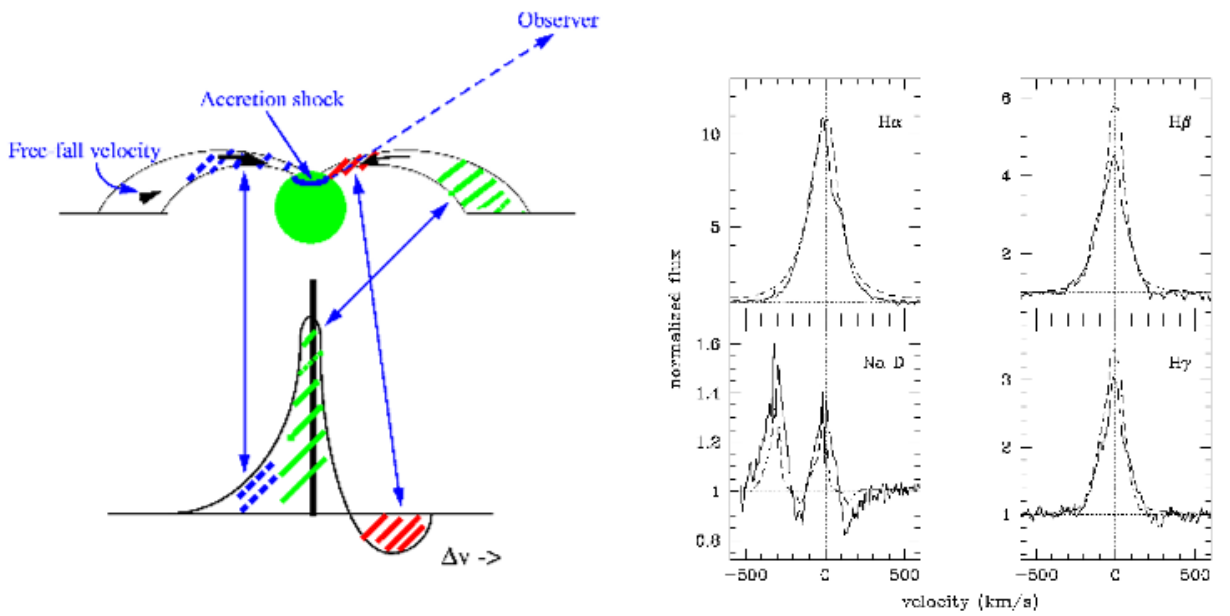

Figure 3. (a) Geometry of magnetospheric accretion and line emission with respect to a distant observer. (b) Predicted line emission from four different line diagnostics. Figure from [4]. 
of the accretion luminosity $L_{a c c}=\left(G M_{*}\left(\dot{M}_{a}\right) / R_{*}\right)\left(1-R_{*} / R_{t}\right)^{1 / 2}$ where typically $R_{t} \simeq 5 R_{*}[11]$. This picture nicely explains the UV excess emission seen from TTSs, which is as noted proportional to the accretion luminosity [12].

The simplified early models of stellar magnetospheres focused on idealized aligned dipole geometries for convenience and ease of calculation. The advent of powerful new spectrographic techniques has completely revolutionized our observational knowledge of the geometry of stellar magnetic fields. The Zeeman Doppler technique utilizes spectropolarimetry to observe magnetically sensitive photospheric lines [14]. Some of these magnetic geometries are rather ordered with a mix of dipole and octopole components. We list 4 of these: AA Tau [15]; BP Tau [16], V2129 Oph [17], and TW Hya [18]. The values of the dipole field strengths (in kGuass) and the dipole to octopole ratios are given as pairs $\left(B_{\text {dip }}, B_{\text {oct }} / B_{\text {dip }}\right)$ for these 4 systems: $(1.7,0.29),(1.2,1.33),(1.0,2.20)$, and $(0.7,4.00)$ respectively [13].

However, much more complex geometries are also observed, as shown for the star V2247 in the left panel of figure 4 . The spectropolarimetry maps provide a knowledge of the footprints of magnetic field lines near the stellar photosphere. By assuming that these stellar fields adopt a current free configuration (otherwise known as potential fields), one can produce maps of the 3D spatial geometry of fields [19-22]. The right panel of figure 4 shows such a structure for V2247 [23]. The structure has a weak dipole component of $<0.1 \mathrm{kGauss}$. The source surface (where the field is open and thus no longer potential is assumed to be 4.6 stellar radii. The top picture of this triptych shows closed field lines associated with an X-ray corona. The middle panel shows the field geometry that most concerns us- the accretion funnel flow. Finally the bottom panel shows open field lines along which a magnetized wind could flow. Simulations of magnetospheric accretion along inclined dipole fields and other geometries are presented by Romanova and her collaborators elsewhere in these proceedings, originating with [24]. The number of funnel streams that flow onto the star in these tipped inclined
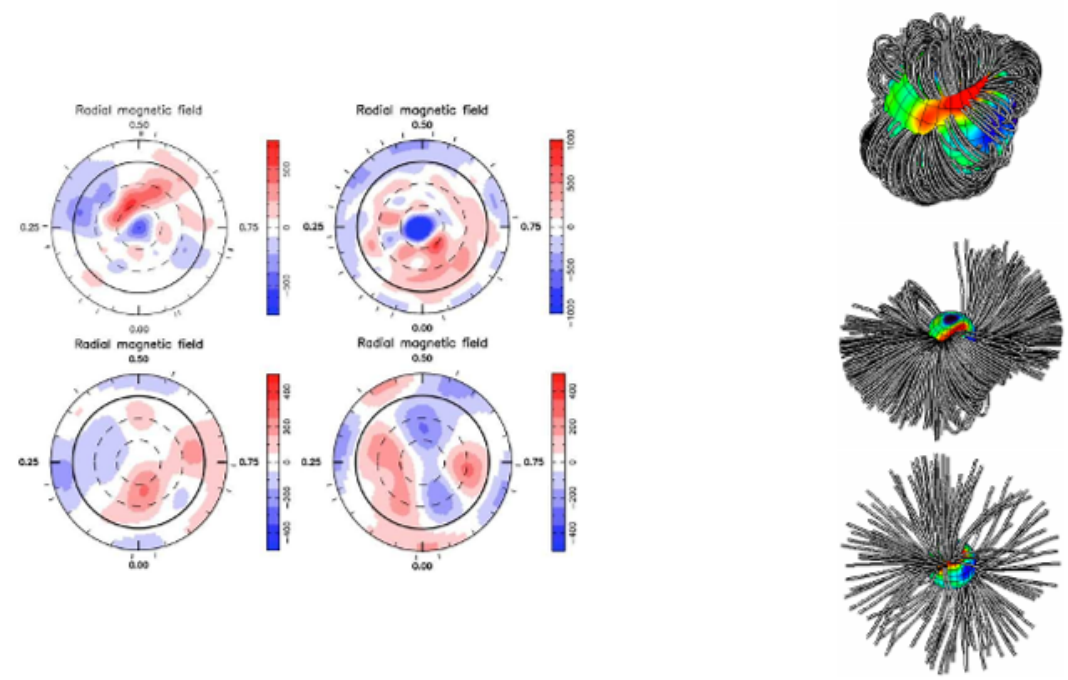

Figure 4. (a) The complex magnetic structure of the surface of V2247 revealed by spetropolarmetric mapping. (b) The magnetic geometry of V2247 as deduced by potential field mapping Adapted from [13]. 


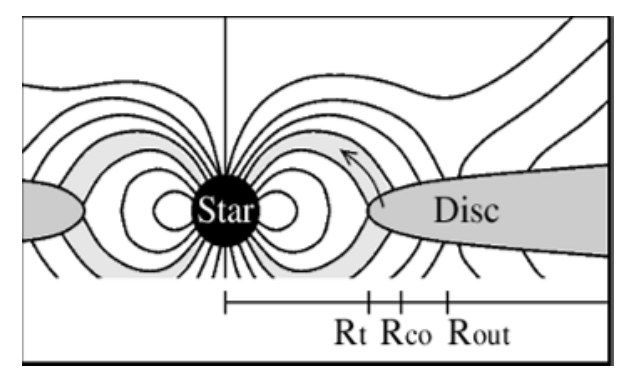

Figure 5. The coupling of a stellar magnetic field to a surrounding disk. The disk is truncated at a few stellar radii, $R_{t}$. The radius at which the Kepler angular frequency of the disk equals that of the star is the co-rotation radius $R_{c o}$. At some distance beyond this, any original stellar field line that is wound to the point that its toroidal field $B_{\phi} \simeq B_{z}$, inflates open and disconnects from the star.

field geometries depends upon the inclination angle: two are found for $\theta<30^{\circ}$, several streams for $30^{\circ}<\theta<60^{\circ}$, and 2 again for larger inclination angles. The structure of the target zone of these streams on the photosphere also varies with inclination - rings of polar emission are observed for low inclinations while bar like emission occurs for larger inclinations.

\section{Counteracting Spinup: Disk Locking?}

How does this interacting stellar magnetosphere / disk system manage the flow of angular momentum? If the accretion stream(s) transports the angular momentum that the gas had at the disk truncation radius, then the rate at which it is deposited on the star produces the spin up accretion torque: $\tau_{a}=\dot{J}=\dot{M} \sqrt{G M_{*} R_{t}}$. This needs to be balanced by a spin-down torque in order to account for the extensive population of low spin PMS. One of the first theoretical models of such a possible mechanism envisaged that magnetic field lines in the more polar part of a dipole magnetosphere would retain a closed magnetic geometry as they penetrated the disk out to large distances [8]. An important radius in this theory is the co-rotation radius $R_{c o}$ which lies beyond the truncation radius and is that distance at which the local angular velocity of material in the Keplerian disk matches the rotation rate of the star and its associated dipolar field. This is illustrated in figure 5. Field lines penetrating the disk between $R_{t}-R_{c o}$ are dragged forwards by the faster rotating disk and therefore transfer angular momentum to the star. Dipolar field lines that penetrate the disk at larger radii $r>R_{c o}$ move more quickly than the gas, and therefore transfer stellar angular momentum to it.

This idea was applied to protostellar spin by [25] and forms the basis of the "disk locking" picture. For some time, this picture dominated the interpretation of the observations. A key prediction was that stellar spins would be regulated to low spin rates as long as a disk was present. With the eventual disappearance of the disk, the magnetospheric connection would be lost and the stars would spin up due to stellar contraction to the main sequence. Early observational evidence was presented in a series of papers [26-28]. By balancing the spin up accretion torque with this disk locked spin down torque, the equilibrium spin rate of this state can be shown to be

$$
\Omega_{*}^{e q}=C \dot{M}^{3 / 7}\left(G M_{*}\right)^{5 / 7} \mu^{-6 / 7}
$$

where $\mu=B_{*} R_{*}^{3}$ and a scale factor $\mathrm{C}$ is assumed to be constant and takes the value $C \simeq 1$. A prediction of the model is that ordered dipole fields need to be strong, $B_{*} \simeq 1 \mathrm{kGauss}$.

Are these theoretical assumptions reasonable? A key aspect of the picture is that a suffienctly large region of the disk beyond $R_{c o}$ be threaded by closed stellar field lines so that the spin of the star can be conveyed into the disk where the disk viscosity transports it away. Note that a dipolar field's energy density dies off with disk radius as $r^{-6}$ so to have a strong field at large radii one must wind up the threading field hundreds if not thousands of times to pump up the local magnetic energy and torque. Is it physically possible to twist a field to this extent? 


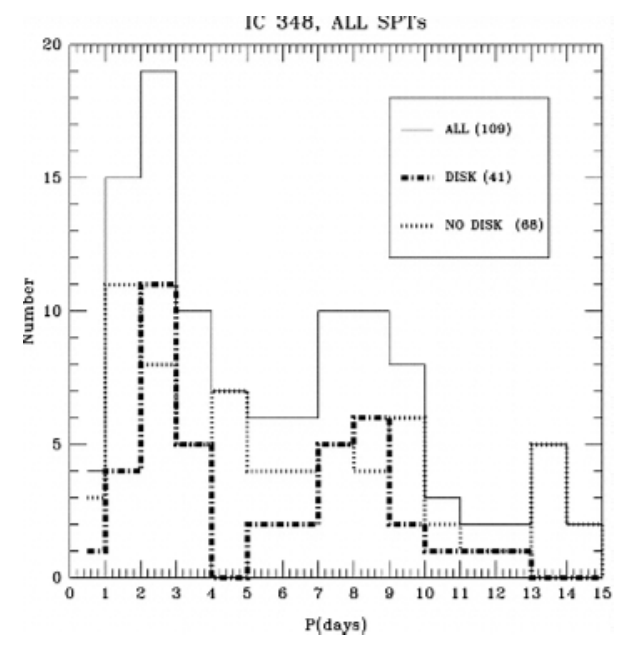

Figure 6. Distribution of stellar spin periods for low and high mass stars with disks detected by Spitzer observations of the young cluster IC348. Adapted from [33].

The answer appears to be no. In calculations started by [29], it can be shown that the wrapping up of a z component of the invading field $B_{z}$ to create a toroidal component $B_{\phi}$ only gets to a modest value of order of the value of $B_{z}$ before the field line inflates and then breaks open. This destroys the connection with the central star and with it, the spin down torque. The same conclusion arises from numerical simulations. Work by $[30,31]$ shows that an initial dipole field that threads the disk will soon be inflated and open up as the strong Kepler shear winds up the penetrating field into a toroidal component which then inflates and opens up beyond a certain radius $R_{\text {out }}$ illustrated in figure 5. Theoretical calculations of [32] showed that the value of this magnetic twist is $\gamma \equiv B_{\phi} / B_{z}=$ $\beta^{-1}\left[\left(r / R_{c o}\right)^{3 / 2}-1\right]$ where the degree of coupling of the magnetic field in the turbulent gas (with a turbulent intensity $\left.\alpha \simeq 10^{-2}\right)$ of the accretion disk is $\beta=(\alpha / \operatorname{Pr})(h / r) \simeq 10^{-2}$. The coupling of the field to the disk, measured by $\beta^{-1}$, is strong: for expected Prandtl numbers of the order of $P r \simeq 1$, both the disk viscosity and the disk aspect ratio $(\mathrm{h} / \mathrm{r})$ together give a value of less than $\beta \simeq 10^{-2}$

The solution of these equations is that only a very small region beyond the co-rotation region is available for a spin down torque to develop. Outside this region, the field lines would be too highly twisted, and thus are opened up by this unavoidable inflationary process. The spin equilibrium period can then be computed in a way that includes this effect. It follows the scaling given above except that now the scale factor $\mathrm{C}$ becomes a function of the magnetic coupling $C(\beta)$. For characteristic values, $C \simeq 10$ and the spin periods become an order of magnitude shorter than is observed. Theoretically at least, stars would all be rotating very quickly.

One of the predictions for the disk locking picture is that there should be a strong observed correlation of disk indicators with slow spin. This measurement depends on the ability to detect both infrared emission from disks as well as stellar spins to high accuracy. The Spitzer space observatory enabled infrared photometry of PMS to detect the presence of disks to unprecedented accuracy. In a major survey of 143 stars in the forming young star cluster IC348, [33] found that there was no correlation between these two quantities. Figure 6 shows that systems with strong or weak indicators of disks have similar spin distributions. Subsequent work by these authors published in 2007 finds that a trend may exist in the Orion region among the more massive stars $\left(>0.4 M_{\odot}\right)$.

Magnetic field measurements also constrain the models [34-37]. As an example, in studies of BP $\mathrm{Tau}$, the Ti I line measurements show that the star has a mean field of $2.8 \mathrm{kG}$. However, spectropolarimetry measurements reveal that there is an upper limit of $200 \mathrm{G}$ on the dipole field strength [38]. Such a weak dipole field presents great difficulties for disk locking pictures. 


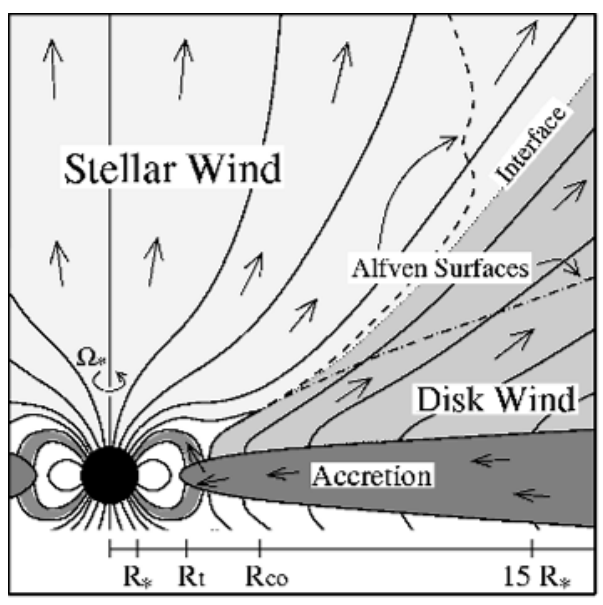

Figure 7. The general picture of an accreting magnetized disk and central magnetized star. Magnetospheric accretion along stellar field lines powers an accretion powered stellar wind. At larger radii, a magnetized disk wind drives a slower wind and jet, involving disk field lines - which is a consequence of the collapse of the magnetized dense core in a molecular cloud. The two winds interact along the interface through pressure forces. Episodic eruptions and activity originate at the magnetosphere/disk boundary as well as along this surface. Adapted from [43].

A second kind of disk-locking theory arises in the context of the X-wind model of [39, 40]. Here the accretion disk is assumed to bottle up most of the magnetic flux of the stellar field. Some of the stellar field lines that are trapped at the inner edge of the disk are assumed to open up and this region is regarded as being the ultimate source of protostellar jets. This outflow is also hypothesized to carry off all of the angular momentum that would otherwise be destined for the star. There is no net accretion torque on the star itself. Thus accreting matter is stripped of its angular momentum near the X-point on the disk with the $\mathrm{X}$-wind carrying this off and away from the system in a jet. The solutions require that $R_{c o} \simeq R_{t}$. This mechanism also requires the presence of strong dipole fields (a few kGauss). The scaling of the equilibrium spin period is also in accord with equation 1 - with a constant $C_{X w i n d}^{e q} \simeq 1$.

An important observational constraint on this model is on the amount of angular momentum that the $\mathrm{X}$-wind carries. By definition, the source of the $\mathrm{X}$-wind is at $R_{c o}$ which is within a few stellar radii. The amount of angular momentum carried by protostellar jets at large distances (20 AU and more) has been determined by HST observations (see review [41]). Using the conservation law, it is possible to determine from what scale on the disk the angular momentum observed in the spinning jet would have originated. The answer is a few AU [42].

\section{Accretion Powered Stellar Winds and Transient Ejecta}

Magnetized winds from central rotors such as main sequence magnetized stars, pulsars, and disks are known to provide powerful spin down torques. The reason basically is that winds can have very long lever arms which makes them powerful brakes even in the absence of powerful magnetic fields. The most natural assumption about the fate of angular momentum at $R_{t}$ is that it is largely carried along with the accreting gas onto the central star, spinning it up. In the absence of disk locking, the only recourse left for it is to be carried off in a stellar magnetized wind along the largely open field lines of a dipole, or higher multipole magnetic fields. What makes these outflows so powerful is the abundant accretion power that is released at the photosphere by the accretion shock. This is many orders of magnitude more power than is available to power an ordinary stellar wind such as our Sun. The basic elements of this theory [43] were developed into a complete theoretical model [44] and extensive numerical simulations [45].

The elements of a general theoretical framework involving both disk and stellar winds as well as magnetoshperic interaction are illustrated in figure 7. There are four basic components. At large disk radii beyond the interaction region is a disk wind whose threading magnetic field lines were part of the 


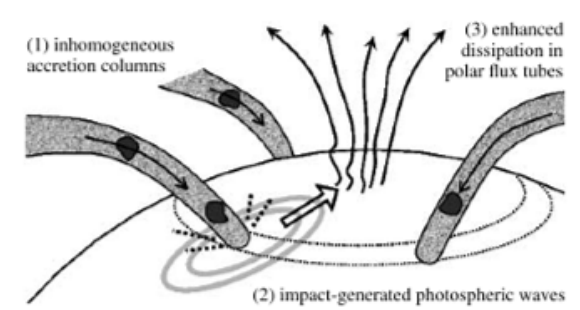

Figure 8. The launch of an accretion driven stellar wind as a consequence of intense magnetic wave generation due to inhomogenous infall along the accretion funnel. Waves propagating away from the impact points and accretion shocks shake up the open field lines and transfer accretion power to an outward bound wave flux. Adapted from [51].

formation of the disk through the original collapse of a magnetized molecular cloud core (for a review see [46]). Magnetospheric accretion brings both gas and its angular momentum to the star where angular momentum is deposited and gravitational potential energy released. The third component is the accretion powered stellar wind carries off the accreted disk angular momentum. Finally, there is the interaction region between the stellar and disk winds. This interface between the disk and the magnetosphere is known from simulations to be highly time variable as accretion proceeds in bursts followed by the inflation of field lines $[47,48]$. The interface region extends up into the wind zones the pressure of the accretion powered stellar wind (APSW) exerts a pressure on the surrounding disk wind, which prevents it from collimating as much as it might in its absence [49]. The entire outflow seen at much larger distances then would contain a high speed core surrounded by a more slowly moving cocoon, which is the collimated disk wind. The eruptions or mass ejections at the interface would add some time dependent behaviour to the jet. The jet can also be heated by a system of shock waves that excite the observed forbidden line emission [50].

The spin down torque upon the star due to the APSW is simply written as $\tau_{w}=\dot{M}_{w} \Omega_{*} r_{A}^{2}$ where $r_{A}$ is the Alfvén radius of the stellar wind. In the theory of disk winds, the wind mass loss rate in disk wind theory is roughly a tenth of the observed accretion rate - a result that is substantiated by many numerical simulations [46]. The same kind of scaling is adopted for APSW winds. The lever arm of the disk wind is the Alfvén radius and must be carefully evaluated. [45] found that standard scalings of 1D models of this radius are unreliable. Their numerical calculations of 2D stellar magnetized winds assumed an initial dipole field and no disk or surrounding disk wind, for a variety of stellar magnetic field strengths, and found that for a $2 \mathrm{kG}$ field, a large Alfvén radius is obtained with $R_{A} \simeq 19.3 R_{*}$. The fraction of the accretion power that is converted into wind power $(\epsilon)$ is one of the main parameters of the APSW theory. Equilibrium solutions for the spin can be found for various objects and this fixes the efficiency parameter. For a strong stellar field of $2 \mathrm{kG}$, the efficiency can be as low as $17 \%$ but this value must be higher for weaker dipole fields. The ratio of the wind torque to the maximum value of a possible disk lock torque is very large, scaling as $\tau_{w} / \tau_{\text {disklock }} \simeq 6.8 \times 10^{4}\left(R_{*} / R_{A}\right)^{2.55}$ so that with the largest Alvén radii for the accretion powered winds, this ratio is over $10^{3}$.

All of this begs the question of how exactly is accretion power transferred into wind power in these models. In other words, what is the theory for the efficiency $\epsilon$ ? It might be expected that a significant amount of the energy released in the shock would be converted into the heating of the gas. This was 
examined by [52] where it was found that at the densities present in the wind, the radiative cooling would be far too efficient for this to act as a pressure source for driving the wind. Consequently these authors predicted that the accretion shock energy must be efficiently coupled to an Alfvén wave driven wind of some kind. Since the wind can thus be relatively cool, this could be a far less "lossy" mechanism. Models of this kind have a distinguished pedigree - wave driven winds for late type stars have been explored by [53-55].

The key idea is that the shaking up of the stellar photosphere in the neighbourhood of the accretion column would launch waves propagating over the photosphere. Those regions with open magnetic field lines would be strongly excited leading to the launch of Alvén waves, as illustrated in figure 8 . This scenario was worked out in some detail in two papers by $[51,56]$. The accretion column is likely to consist of fairly lumpy gas. The energy released by each of these clumps in the inghomogenous accretion column is roughly $10 \%$ of the kinetic energy of the clump multiplied by the ratio of the clump density to the background flow. Therefore, for strong cooling and instability, significant power is available to create the photospheric wave flux. Comparison of the model results to 14 stars in the Taurus Auriga region shows some interesting results: there is evidence for clumpy accretion from the level of hourly variability of these sources. The predicted mass loss rates are slightly lower than observed, and there is a good link with X-ray observation of shocks.

An important aspect of the coronal mass ejections (CMEs) that occur at the magnetosphere/disk interface is that they could carry significant amounts of angular momentum. Field lines connecting the star to the inner edge of the disk will, as we have argued, become twisted and inflate. These will then pinch off and be released much like coronal ejections from the Sun [48]. The ejections have been shown to exert a considerable torque on both the inner disk as well as the star - in the latter case as long as the CME is more slowly rotating than the star. These effects may reduce the net spin up torque upon the star, making the efficiency constraints needed to power the stellar wind less severe. Clearly more high resolution 3D numerical simulations will be needed to explore these important issues much more deeply.

In conclusion major observational progress has been made in several respects; unprecedented spectrophotometric imaging, measurements of rotations of thousands of stars, X-ray and other observations of rapid variability, and magnetospheric mapping, Similar progress has been made on the theoretical side: new and more comprehensive theoretical models, detailed 2D and 3D simulations, time variability, and the ability to match models with data in considerable depth. We have learned that magnetosphere/disk coupling is unlikely to work by disk locking. Rather accretion powered stellar winds together with CMEs appear to be at work. More progress requires additional concrete evidence for accretion powered stellar winds while on the theoretical side, the complex structure of stellar magnetic fields has yet to be fully integrated into the theory.

\section{References}

[1] W. Herbst, C.A.L. Bailer-Jones, R. Mundt, K. Meisenheimer, R. Wackermann, Astronomy and Astrophysics 396, 513 (2002)

[2] W. Herbst, J. Eislöffel, R. Mundt, A. Scholz, Protostars and Planets V p. 297 (2007)

[3] J. Bouvier, S.P. Matt, S. Mohanty, A. Scholz, K.G. Stassun, C. Zanni, PPVI proceedings (2013)

[4] J. Muzerolle, N. Calvet, L. Hartmann, The Astrophysical Journal 550, 944 (2001)

[5] L. Hartmann, E.H. Avrett, R. Loeser, N. Calvet, Astrophysical Journal 349, 168 (1990)

[6] R. Kurosawa, T.J. Harries, N.H. Symington, Monthly Notices of the Royal Astronomical Society 370, 580 (2006)

[7] K. Davidson, J.P. Ostriker, Astrophysical Journal 179, 585 (1973) 
[8] P. Ghosh, F.K. Lamb, Astrophysical Journal 223, L83 (1978), a\&AA ID. AAA021.064.080

[9] P.J. Armitage, C.J. Clarke, Monthly Notices of the Royal Astronomical Society 280, 458 (1996)

[10] L. Hartmann, N. Calvet, E. Gullbring, P. D’Alessio, Astrophysical Journal v.495 495, 385 (1998)

[11] N. Calvet, E. Gullbring, The Astrophysical Journal 509, 802 (1998)

[12] E. Gullbring, N. Calvet, J. Muzerolle, L. Hartmann, The Astrophysical Journal 544, 927 (2000)

[13] S.G. Gregory, J.F. Donati, Astronomische Nachrichten 332, 1027 (2011)

[14] J.F. Donati, M.M. Jardine, S.G. Gregory, P. Petit, J. Bouvier, C. Dougados, F. Ménard, A.C. Cameron, T.J. Harries, S.V. Jeffers et al., Monthly Notices of the Royal Astronomical Society 380, 1297 (2007)

[15] J.F. Donati, M.B. Skelly, J. Bouvier, S.G. Gregory, K.N. Grankin, M.M. Jardine, G.A.J. Hussain, F. Ménard, C. Dougados, Y. Unruh et al., Monthly Notices of the Royal Astronomical Society 409, 1347 (2010)

[16] J.F. Donati, M.M. Jardine, S.G. Gregory, P. Petit, F. Paletou, J. Bouvier, C. Dougados, F. Ménard, A.C. Cameron, T.J. Harries et al., Monthly Notices of the Royal Astronomical Society 386, 1234 (2008)

[17] J.F. Donati, J. Bouvier, F.M. Walter, S.G. Gregory, M.B. Skelly, G.A.J. Hussain, E. Flaccomio, C. Argiroffi, K.N. Grankin, M.M. Jardine et al., Monthly Notices of the Royal Astronomical Society 412, 2454 (2011)

[18] J.F. Donati, S.G. Gregory, S.H.P. Alencar, J. Bouvier, G. Hussain, M. Skelly, C. Dougados, M.M. Jardine, F. Ménard, M.M. Romanova et al., Monthly Notices of the Royal Astronomical Society p. 1284 (2011)

[19] M. Jardine, K. Wood, A.C. Cameron, J.F. Donati, D.H. Mackay, Monthly Notice of the Royal Astronomical Society 336, 1364 (2002)

[20] M. Jardine, A.C. Cameron, J.F. Donati, S.G. Gregory, K. Wood, Monthly Notices of the Royal Astronomical Society 367, 917 (2006)

[21] S.G. Gregory, M. Jardine, I. Simpson, J.F. Donati, Monthly Notices of the Royal Astronomical Society 371, 999 (2006)

[22] S.G. Gregory, S.P. Matt, J.F. Donati, M. Jardine, Monthly Notices of the Royal Astronomical Society 389, 1839 (2008)

[23] J.F. Donati, M.B. Skelly, J. Bouvier, M.M. Jardine, S.G. Gregory, J. Morin, G.A.J. Hussain, C. Dougados, F. Ménard, Y. Unruh, Monthly Notices of the Royal Astronomical Society 402, 1426 (2010)

[24] M.M. Romanova, G.V. Ustyugova, A.V. Koldoba, R.V.E. Lovelace, The Astrophysical Journal 610, 920 (2004)

[25] A. Koenigl, Astrophysical Journal 370, L39 (1991)

[26] S. Edwards, S.E. Strom, P. Hartigan, K.M. Strom, L.A. Hillenbrand, W. Herbst, J. Attridge, K.M. Merrill, R. Probst, I. Gatley, Astronomical Journal (ISSN 0004-6256) 106, 372 (1993)

[27] J. Bouvier, M. Forestini, S. Allain, Astronomy and Astrophysics 326, 1023 (1997)

[28] L.M. Rebull, S.C. Wolff, S.E. Strom, The Astronomical Journal 127, 1029 (2004)

[29] D.A. Uzdensky, A. Königl, C. Litwin, The Astrophysical Journal 565, 1191 (2002)

[30] M.M. Romanova, G.V. Ustyugova, A.V. Koldoba, R.V.E. Lovelace, The Astrophysical Journal 578, 420 (2002)

[31] C. Zanni, J. Ferreira, Astronomy and Astrophysics 508, 1117 (2009)

[32] S. Matt, R.E. Pudritz, Monthly Notices of the Royal Astronomical Society 356, 167 (2005)

[33] L. Cieza, N. Baliber, The Astrophysical Journal 649, 862 (2006) 
[34] G. Basri, G.W. Marcy, The Astrophysical Journal 431, 844 (1994)

[35] E.W. Guenther, Herbig-Haro Flows and the Birth of Stars; IAU Symposium No. 182 182, 465 (1997)

[36] C.M. Johns-Krull, J.A. Valenti, C. Koresko, The Astrophysical Journal 516, 900 (1999)

[37] D.A. Smirnov, S.A. Lamzin, S.N. Fabrika, G.A. Chuntonov, Astronomy Letters 30, 456 (2004)

[38] C.M. Johns-Krull, J.A. Valenti, A.P. Hatzes, A. Kanaan, The Astrophysical Journal 510, L41 (1999)

[39] F. Shu, J. Najita, E. Ostriker, F. Wilkin, S. Ruden, S. Lizano, The Astrophysical Journal 429, 781 (1994)

[40] S. Mohanty, F.H. Shu, The Astrophysical Journal 687, 1323 (2008)

[41] T. Ray, C. Dougados, F. Bacciotti, J. Eislöffel, A. Chrysostomou, Protostars and Planets V p. 231 (2007)

[42] J.M. Anderson, Z.Y. Li, R. Krasnopolsky, R.D. Blandford, The Astrophysical Journal 630, 945 (2005)

[43] S. Matt, R.E. Pudritz, The Astrophysical Journal 632, L135 (2005)

[44] S. Matt, R.E. Pudritz, The Astrophysical Journal 681, 391 (2008)

[45] S. Matt, R.E. Pudritz, The Astrophysical Journal 678, 1109 (2008)

[46] R.E. Pudritz, R. Ouyed, C. Fendt, A. Brandenburg, Protostars and Planets V p. 277 (2007)

[47] A.P. Goodson, R.M. Winglee, The Astrophysical Journal 524, 159 (1999)

[48] C. Zanni, J. Ferreira, Astronomy \& Astrophysics 550, 99 (2013)

[49] C. Fendt, The Astrophysical Journal 692, 346 (2009)

[50] J.E. Staff, B.P. Niebergal, R. Ouyed, R.E. Pudritz, K. Cai, The Astrophysical Journal 722, 1325 (2010)

[51] S.R. Cranmer, The Astrophysical Journal 706, 824 (2009)

[52] S. Matt, R.E. Pudritz, Star-Disk Interaction in Young Stars 243, 299 (2007)

[53] L. Hartmann, K.B. MacGregor, Astrophysical Journal 242, 260 (1980)

[54] W.M. Decampli, Astrophysical Journal 244, 124 (1981), a\&AA ID. AAA029.121.014

[55] T.K. Suzuki, S. ichiro Inutsuka, The Astrophysical Journal 632, L49 (2005)

[56] S.R. Cranmer, The Astrophysical Journal 689, 316 (2008) 
